\title{
TRANSURETHRAL RESECTION OF PROSTATE (TURP): SURGERY WITHOUT USING A 3-WAY CATHETER
}

Adam Jones ${ }^{1}$, Syed Ali Ehsanullah ${ }^{1}$, Amerdip Birring ${ }^{1}$, Connor Gascoigne ${ }^{2}$, Sid Singh ${ }^{1}$, Andrea Ginepri ${ }^{1}$, Ike Apakama $^{1}$

${ }^{1}$ George Eliot Hospital, Nuneaton, United Kingdom

${ }^{2}$ University of Leicester, Leicester, United Kingdom

Corresponding author Adam Jones: apjones47@doctors.org.uk

Submitted: May 4, 2018. Accepted: July 7, 2018. Published: September 24, 2018.

Transurethral resection of the prostate (TURP) continues to represent the standard surgical management of benign prostatic hyperplasia (BPH). ${ }^{1}$ The success of this commonly performed procedure is suggested by significant improvements in patient symptom scores, flow rate studies and post-void residual (PVR) volumes. ${ }^{2}$ Despite this, TURP continues to be associated with multiple complications, consisting mainly of post-operative bleeding requiring transfusion, prolonged use of indwelling catheter, transurethral resection (TUR) syndrome, urinary incontinence and erectile dysfunction (ED).

Insertion of 3-way urethral catheters and use of continuous bladder irrigation (CBI) is frequently used following endoscopic urological procedures. It is common practice to insert a 3-way catheter following a TURP in a bid to prevent intravesical clot formation. Frequently, large catheters (22-24Fr) with high volume $(30 \mathrm{~mL})$ anchoring balloons are used to provide traction to ensure haemostasis. ${ }^{3}$

Using 3-way catheters and CBI reduces often leads to longer length of post-operative catheterization and ultimately increased length of stay. The routine use of a 3-way is of substantial cost to the healthcare provider in comparison to 2-way catheters. A leading UK medical supplier prices a 3 -way at $£ 9.28$ (22Fr) and $£ 10.44$ (24Fr), which is significantly more expensive than a comparable sized 2-way catheter at $£ 1.48(22 \mathrm{Fr}$ PTFE) or $£ 3.76$ (Hydrogel 22Fr). ${ }^{4}$
We believe that our use of inserting 2-way catheters following TURP is unique. We aim to report comparable rates of post-operative complications to those reported in the literature, and to establish if 2-way urethral catheterization is a safe option for the patients at reduced cost to the health care provider by reducing length of stay.

\section{METHODOLOGY}

\section{Study Design}

All patients scheduled to undergo TURP at our institution were enrolled into this prospective cohort study. There was no particular selection criterion for those involved and prostate size or pathology (benign or malignant) was not considered. Those excluded from the study were patients with confirmed or uncorrected bleeding disorders.

Size 22-Fr PTFE 2-way catheters were placed, with the balloons inflated with $30 \mathrm{~mL}$ of sterile water following TURP. Either a consultant or registrar under consultant supervision would perform the operation using bipolar diathermy with $0.9 \%$ saline as irrigation fluid. All patients would have trial without catheter following review by the urology team the following morning if suitable.

The variables recorded included length of stay, length of catheterization days, grams resected, and pre-operative and post-operative hemoglobin. The study also looked at post-operative complications 
that may be associated with its use, such as significant hematuria, clot retention, TUR syndrome and urinary tract infection.

It was thought that this would also provide an opportunity to assess the functional outcome data at our institution. We therefore included pre-operative and post-operative Qmax, PVR, international prostate symptom scores and symptom-bother scores 3 months following TURP.

The primary outcome is to assess if 2-way catheters are safe following bipolar-TURP. The purpose is to assess if this substantive change in practice can allow cost-savings associated with the use of less expensive 2 -way catheters and reduction in length of stay.

\section{LITERATURE REVIEW}

To evaluate our primary outcome for this study, it was important to compare our outcomes of a 2-way as compared to those of a 3-way catheter. To our knowledge, there is no peer-reviewed article in the literature regarding 2-way urethral catheters following TURP. The only evidence we could find was a non-peerreviewed abstract by Kapasi et al. ${ }^{5}$ They concluded that 3-way are superior to 2-way catheters in a cohort of 48 patients who underwent TURP. However, the TURP was performed using a monopolar technique and no rationale or data was available to support their conclusions.

Al-Rawashdah et al., reported peri-operative outcomes between monopolar and bipolar-TURP. ${ }^{6}$ This was the most recent study on PubMed search, and was adequately powered with statistically significantly better peri-operative outcomes of patients in the bipolar TURP group. All patients received a 3-way urethral catheter following TURP. The outcomes of bipolarTURP in this above publication, which we chose for comparison, were similar to other publications on this topic.

\section{APPROVAL AND GOVERNANCE}

The study was registered with the clinical audit department and has followed the approved process within the George Eliot hospital. As part of this, the project team agrees that this study is in line with the department's ethical principles and with consideration to issues regarding governance and confidentiality.

\section{STATISTICAL ANALYSIS}

Statistical analysis was performed with Chi-squared test using R-Studio programme.

\section{RESULTS}

A total of 143 consecutive patients underwent bipolar-TURP between October 2015 and July 2017. The indications for TURP were BPH (129/143) causing severe LUTS refractory to medical therapy, acute urinary retention or use of long-term catheter, those with prostate cancer (4/143) and re-do TURPs $(10 / 143)$.

The mean age of the patients included in this study was 71.9 (52-89) with a mean PSA of $11.2 \mathrm{ng} / \mathrm{mL}$. There were $7 / 143(4.9 \%)$ patients taking warfarin prior to TURP, there was one patient taking rivaroxaban $(0.7 \%)$. All these patients were taking anticoagulation for atrial fibrillation and had an INR $<1.4$ prior to surgery. Patient demographics are outlined in Table 1.

Following resection, 132/143 (92.3\%) patients had 22-Fr 2-way catheters with $30 \mathrm{ml}$ sterile water to inflate the balloon. There were 11/143 (7.7\%) patients who had a 3-way placed for CBI. Patients receiving 3 -way catheters from our study were not included in our results. This was because patients with a 3-way could not contribute to the primary objective of the study.

Patients with 3-way catheters post-operatively had size 22-Fr and had CBI. Of these, 8/11 had TURP performed by the same consultant who was new to the department. The remaining 3 patients had documented heavy intraoperative hemorrhage during resection.

Patients had regional anesthesia as a spinal anesthetic $(71 / 132,53.8 \%)$, or general anesthetic $(61 / 132$, $46.2 \%)$. The mean resection time was 44.8 minutes (range 10-100), which was less (68.3 minutes) than reported in the current literature by Al-Rawashdah et al. ${ }^{6}$ This is in keeping with a reduced resected specimen weight of 22.8 grams (2-way) versus 34.5 grams (3-way). ${ }^{6}$ Intraoperative details are listed in Table 2.

Post-operatively, there was reduced mean length of stay (1.45 versus 3.27 days) and length of catheterization days ( 1.24 versus 3.13$)$. The maximum length of 


\begin{tabular}{|l|c|c|}
\hline TABLE 1 Patient Demographics, Pre-operative Variables \\
\hline Variable & 2-way (Our Study) & 3-way (Current Literature) \\
\hline Patients (n) & 143 & 246 \\
\hline Age mean (range) & $71.9(52-89)$ & 67.72 \\
\hline ASA median (range) & $2(1-3)$ & \\
\hline Anti-Coagulants & $7 / 143(5.0 \%)$ & \\
\hline Warfarin & $1 / 143(0.7 \%)$ & \\
\hline Rivaroxaban & $18 / 143(12.5 \%)$ & \\
\hline Anti-platelets & $4 / 143(2.8 \%)$ & \\
\hline Aspirin & $129 / 143(90.5 \%)$ & 14.34 \\
\hline Clopidogrel & $4 / 143(2.8 \%)$ & \\
\hline Indication & $10 / 143(6.7 \%)$ & \\
\hline BPH (LUTS, AUR, LTC) & 11.2 & \\
\hline Prostate Cancer & & \\
\hline Re-do TURP & \multicolumn{2}{|c|}{} \\
\hline Serum PSA (mean) & \\
\hline Current literature: 3-way catheter use following bipolar-TURP - Al-Rawashdah S et al. ${ }^{6}$ \\
\hline
\end{tabular}

\begin{tabular}{|c|c|c|}
\hline \multicolumn{3}{|c|}{ TABLE 2 Intraoperative Details } \\
\hline Variable & 2-way (Our study) & 3-way (Current Literature) ${ }^{1}$ \\
\hline \multicolumn{3}{|l|}{ Anesthetic } \\
\hline Spinal & $71 / 132(53.8 \%)$ & \\
\hline General Anaesthetic & $61 / 132(46.2 \%)$ & \\
\hline \multicolumn{3}{|c|}{ Resection Time (minutes) } \\
\hline Mean & 44.8 & 68.3 \\
\hline Range & $10-100$ & \\
\hline \multicolumn{3}{|c|}{ Theatre Specimen Weight (g) } \\
\hline Mean & 22.8 & 34.5 \\
\hline Range & $2.0-70.0$ & \\
\hline \multicolumn{3}{|l|}{ Diathermy } \\
\hline Monopolar & $0 / 132(0.0 \%)$ & $0 / 246(0.0 \%)$ \\
\hline Bipolar & $132 / 132(100.0 \%)$ & $246 / 246(100.0 \%)$ \\
\hline \multicolumn{3}{|l|}{ Catheter } \\
\hline 2-way & $132 / 143(92.3 \%)$ & $0 / 246(0.0 \%)$ \\
\hline 3-way & $11 / 143(7.7 \%)$ & $246 / 246(100 . \%)$ \\
\hline
\end{tabular}

J Endolum Endourol Vol 1(2):e3-e9; September 24, 2018.

This article is distributed under the terms of the Creative Commons Attribution-Non Commercial 4.0 International License. (c) 2018 Jones et al. 
stay was 4 days; this was due to post-operative bleeding that required transfusion of 2 units of packed red cells. This represented the largest drop in hemoglobin at $3.4 \mathrm{~g} / \mathrm{dL}$.

The mean drop in hemoglobin levels for 2-way catheters was $0.7 \mathrm{~g} / \mathrm{dL}$. This was less than patients with a 3-way reported by Al-Rawashdah et al. (1.63 $\mathrm{g} / \mathrm{dL}$ ). Despite this, the rate of hematuria requiring blood transfusion was higher in our 2-way catheter cohort $(2 / 132,1.5 \%$ versus $0 / 246,0.0 \%, p=0.05$; $95 \% \mathrm{CI}=0.3509$ ). However, there were no patients who had to return to theatre as a result of significant hemorrhage.

Urinary tract infection was higher in our study $(15.2 \%$ versus $8.2 \%)$. There were no patients with TUR syndrome $(0 / 132,0.0 \%)$. At 3 months postoperatively, there were $2 / 132(1.5 \%)$ of patients with ED and 7/132 (5.3\%) with incontinence. Post-operative complications are outlined in Table 3.
Functional outcomes pre-operatively and postoperatively were favourable following bipolar-TURP at our centre. This included significant improvement in IPSS scores pre- and post-operatively (20 versus 6.9), as well as improved Qmax and PVR residual scans (Table 4).

\section{DISCUSSION}

Following TURP, 3-way urethral catheters are inserted for CBI in a bid to prevent clot retention. The aim of this study was to assess if 2-way catheters could be used at reduced cost to the health provider by evaluating the safety and efficacy of this approach. Our rationale was the anchoring effect of the urethral catheter balloon under traction could provide sufficient hemostasis post-operatively, and allow for earlier catheter removal day 1 post-operatively.

There are around 15,000 TURPs are performed in the NHS each year. The cost of this procedure is

TABLE 3 Post-operative Complications

\begin{tabular}{|l|c|c|c|}
\hline Variable & 2-way (Our study) & 3-way (Current Literature) & p-value \\
\hline Significant Hematuria & $2 / 132(1.5 \%)$ & $0 / 246(0.0 \%)$ & 0.05 \\
\hline Clot retention & $5 / 132(3.9 \%)$ & $4 / 246(1.62 \%)$ & 0.18 \\
\hline TUR syndrome & $0 / 132(0.0 \%)$ & $0 / 246(0.0 \%)$ & $\mathrm{n} / \mathrm{a}$ \\
\hline UTI & $20 / 132(15.2 \%)$ & $21 / 246(8.2 \%)$ & 0.05 \\
\hline Erectile Dysfunction & $2 / 132(1.5 \%)$ & Not reported & $\mathrm{n} / \mathrm{a}$ \\
\hline Incontinence & $7 / 132(5.3 \%)$ & Not reported & $\mathrm{n} / \mathrm{a}$ \\
\hline Current Literature: 3-way catheter use following bipolar-TURP - Al-Rawashdah S et al. & \\
\hline
\end{tabular}

\section{TABLE 4 Functional Outcomes}

\begin{tabular}{|l|c|c|c|c|}
\hline Variable & Pre-op 2-way & Post-op 2-way & Pre-op 3-way $^{\mathbf{1}}$ & Post-op 3-way $^{2}$ \\
\hline Qmax & $9.7(1-19)$ & $21.6(5.2-6.7)$ & 8.69 & 20.84 \\
\hline PVR & $218.2(0-1200)$ & $62(0-316)$ & 93.09 & 25.12 \\
\hline IPSS & $20(5-34)$ & $6.9(0-29)$ & 4.63 & 1.51 \\
\hline Bother score & $3.6(2-6)$ & $1.47(0-6)$ & - & - \\
\hline${ }^{*}$ Our Study & & & \\
\hline${ }^{* *}$ Current Literature: Al-Rawashdah S, et al. ${ }^{6}$ & \\
\hline
\end{tabular}


around $£ 2,961$, and represents a significant burden to NHS resources. Efforts can be made to reduce this cost by decreasing length of stay. A key driver for this is using a 2-way instead of a 3-way catheter without the use CBI by allowing earlier catheter removal.

The cost of a 2-way is also less than a 3-way catheter. A leading UK medical supplier prices a 3 -way at $£ 9.28$ (22-Fr) and a 2 -way catheter at $£ 1.48$ (22-Fr PTFE). ${ }^{4}$ This allows for a small cost saving of $£ 7.80$, which in the context of the cost of the operation $(£ 2,961)$ is not significant. However, the average cost of a hospital bed in the NHS is around $£ 222$ per day. $^{7}$ a shorter length of stay associated with 2-way catheters will allow for greater cost-savings.

There was a reduced mean fall in hemoglobin level in our 2-way catheter group than reported in literature for 3-way catheters used for comparison $(0.6 \mathrm{~g} / \mathrm{dL}$ versus $1.63 \mathrm{~g} / \mathrm{dL}$ ). This may be because the surgeon is more assiduous in his intraoperative hemostasis; this comes from a heightened awareness that they are not using post-operative irrigation through a 3-way catheter.

There were 5/132 (3.9\%) patients that required conversion of 2-way to 3-way catheters due to hematuria and clot retention. Three of these patients had more grams resected in comparison to the cohort mean (60 g, $40 \mathrm{~g}, 44 \mathrm{~g}$ versus $22.8 \mathrm{~g}$ ). There was also no significant difference in post-operative complications for patients receiving either 2-way or 3-way catheters when comparing our results to the chosen literature. ${ }^{6}$

Patients undergoing TURP at our centre had a mean stay of 1.45 days. All suitable patients had their catheters removed the following morning, and $101 / 132(76.5 \%)$ had a successful removal day 1 postoperatively. The remaining 31/132 had their catheter replaced and planned removal in the urology unit. This results in cost-savings to the healthcare provider. A recent "Getting It Right First Time (GIRFT)" review of the department commended us for this length of stay being amongst the lowest in urology departments across the country. We are currently looking to shorten this length of stay further by discharging suitable patients on the same day of the TURP.

The limitations of this study include the small sample size used to report the validity of this unique approach. This was because of the short time of 20 months over which the Bipolar-TURPs were performed.
There is a similar trend for patients having a 2-way catheter for TURPs for 10 years before the period of this study. However, because of a lower level of validity of this historical data, we have not included them in our study.

Further, there is no published article or peer-reviewed data on this approach. As this is now standard of care in our department, it was not possible to have a comparative group within our study. We, therefore, compared our outcomes to those of a 3-way catheter in contemporaneous literature.

It is pertinent to note that the new consultant who was using 3-way catheters at the start, very rapidly changed his practice of 20 years when he saw the superior outcomes of his fellow consultants. This much-improved change in his clinical practice has been now sustained for the last 18 months. Therefore, we feel that all practicing urologists can easily adopt this change of practice.

\section{CONCLUSIONS}

We provide evidence from our study of a better patient experience and length of stay reduction allowing for cost effectiveness when using a 2-way catheter following bipolar-TURP. It is possible that this technique may be best suited to prostates smaller than 40 grams. For the larger prostates, the new gold standard is now an enucleation rather than resection of the prostate. Thus, for the smaller prostate, we feel that a 2-way catheter bipolar-TURP should become the gold standard, and that this is an easy change in our practice to adopt; one, which is scalable and sustainable. These findings can then be replicated on a larger scale and in multiple centres.

\section{FUNDING AND CONFLICT OF INTEREST}

There was no funding required for this study. There was no conflict of interest from the authors involved.

\section{ACKNOWLEDGEMENTS}

The authors would like to thank Mr Ike Apakama for introducing this novel technique to the department and allowing review of the outcomes. We also thank the reviewer for their comments on an earlier version of the article, which have led to significant changes and improvements to the final article. 


\section{REFERENCES}

1. Rassweiler J, Teber D, Kuntz R et al. Complications of Transurethral Resection of the Prostate (TURP) Incidence, Management, and Prevention. Eur Assoc Urol 2006;50:969-80.

2. Ahyai S, Gilling P, Kaplan S et al. Meta-analysis of functional outcomes and complications following transurethral procedures for lower urinary tract symptoms resulting from benign prostatic enlargement. Eur Assoc Urol 2010;58:384-97.

3. Abdelrahman M, Davis N, McMahon B et al. A comparative assessment of irrigation and drainage characteristic for commercially available urethral catheters. Central Eur J Urol 2017:70:382-87.
4. BARD Medical Supplies. Homepage [Internet]. Available at: www.bardmedical.co.uk/products.

5. Kapasi F, Mufti U, Pandit A et al. Is the use of two way catheter post-TURP safe? UroToday Int J 2008;1.

6. Al-Rawashdah S, Pastore A, Salhi Y et al. Prospective randomized study comparing monopolar with bipolar transurethral resection of prostate in benign prostatic obstruction: 36 month outcomes. World J Urol 2017:35;1595-601.

7. National Institute for Health and Care Excellence; NICE guideline (NG27). Transition between inpatient hospital settings and community or care home settings for adults with social care needs. NICE guidelines 2015/16. 